\title{
Gastric carcinosarcoma: A case report and review of the literature
}

\author{
ROBERTO CIROCCHI $^{1 *}$, STEFANO TRASTULLI $^{1 *}$, JACOPO DESIDERIO $^{1 *}$, \\ VERONICA GRASSI $^{1 *}$, IVAN BARILLARO ${ }^{1}$, ALBERTO SANTORO ${ }^{3 *}$, CARLO BOSELLI $^{4 *}$, \\ NICOLA AVENIA $^{5 *}$, GIUSEPPE NOYA ${ }^{4 *}$ and ANGELO DE SOL ${ }^{1 *}$ \\ ${ }^{1}$ Department of General Surgery, University of Perugia, St. Maria Hospital, Terni; ${ }^{2}$ Pathological Anatomy Unit, \\ University of Perugia, Perugia; ${ }^{3}$ Department of Surgical Sciences, Sapienza University of Rome, Rome; \\ ${ }^{4}$ Department of General and Oncologic Surgery, and ${ }^{5}$ Endocrine Surgical Unit, \\ Department of Surgical Sciences, Radiology and Dentistry, University of Perugia, Perugia, Italy
}

Received November 21, 2011; Accepted April 17, 2012

DOI: 10.3892/ol.2012.699

\begin{abstract}
Carcinosarcoma of the stomach is a rare biphasic tumor that consists of both carcinomatous and sarcomatous components. The common carcinoma component is tubular or papillary adenocarcinoma and the mesenchymal sarcomatous components are variable but may include leiomyosarcoma, rhabdomyosarcoma and osteosarcoma. The aim of this study was to describe the characteristics of gastric carcinosarcoma and to present a review of the available literature. We report a case of carcinosarcoma in a 62-year-old female including the clinical and histopathological features of this tumor. Following ultrasound and computed tomography scans, laparotomy was performed, revealing a large mass, followed by radical surgery. Macroscopically, a polypoid tumor was observed. Microscopically, the tumor was composed of moderately differentiated adenocarcinoma and poorly differentiated sarcoma with a high mitotic index and necrotic areas. At present, the achievement of a definitive diagnosis is dependent on immunohistochemical staining and radical surgery. Thus, more effective diagnostic methods are required to improve patient survival.
\end{abstract}

\section{Introduction}

Carcinosarcoma of the stomach is an extremely rare mixed tumor comprising carcinoma and sarcoma components (1). The most common carcinoma component is tubular or papillary adenocarcinoma, while neuroendocrine carcinomatous differentiation is less common. The mesenchymal sarcoma-

Correspondence to: Dr Jacopo Desiderio, Department of General Surgery, University of Perugia, St. Maria Hospital, Via Tristano di Joannuccio, Terni, I-05100, Italy

E-mail: djdesi85@hotmail.it

${ }^{*}$ Contributed equally

Key words: carcinosarcoma, gastric tumor tous component is variable and may include leiomyosarcoma, rhabdomyosarcoma, osteosarcoma or chondrosarcoma (2).

Tumor in the upper gastrointestinal tract tends to be localized in the esophagus where it represents approximately $3 \%$ of all esophageal tumors. By contrast, only 52 cases of gastric carcinosarcoma have been reported in the literature thus far, most of which are described in the Japanese literature (3). Other terms for this tumor are sarcomatoid carcinoma of the stomach and spindle cell carcinoma of the stomach. The average survival of these patients is 2-6 months. In the present study, we report a case of gastric carcinosarcoma and review some of the literature. Consent was obtained from the patient.

\section{Case report}

A 62 year-old woman was admitted to our division reporting a history of epigastric pain with asthenia and weight loss (8 kilos over 3 months). The physical examination revealed no specific findings. The patient underwent the following laboratory tests and endoscopic examinations: esophagogastroduodenoscopy (EGD), ultrasound (US) and computed tomography (CT) scanning. The EGD revealed an ulcerated mass in the gastric body-fundus.

On abdominal US, three hepatic mass lesions were revealed, the largest one being $5 \times 3 \mathrm{~cm}$ in size. Abdominal CT revealed that the tumor had metastasized through the gastric wall up to the serose and confirmed the hepatic US report. The patient subsequently underwent an exploratory laparotomy that revealed a large mass $(20 \times 15 \mathrm{~cm}$ in size). The tumor had infiltrated the pancreatic body-tail, gastric body fundus, splenic hilium and left adrenal gland. A total gastrectomy with Rouxen-Y esophagojejunostomy with body-tail pancreatic resection and left surrenalectomy was performed.

Despite the poor prognosis, radical surgery was performed since the tumor had invaded most of the organ and perivisceral structures. Radiofrequency ablation (RFA) was used for the treatment of the hepatic lesions.

Macroscopically, an ulcerative polypoid tumor $(13 \times 10 \mathrm{~cm})$ arising from the gastric body fundus was observed. The mass had infiltrated through all layers of the gastric wall and extended to the pancreatic tail. The spleen was not infiltrated 


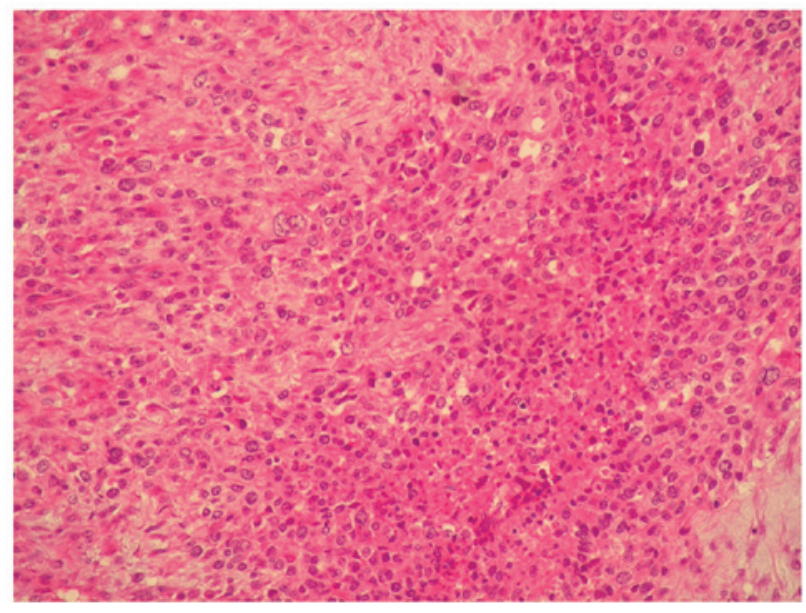

Figure 1. The sarcomatoid component of carcinosarcoma.

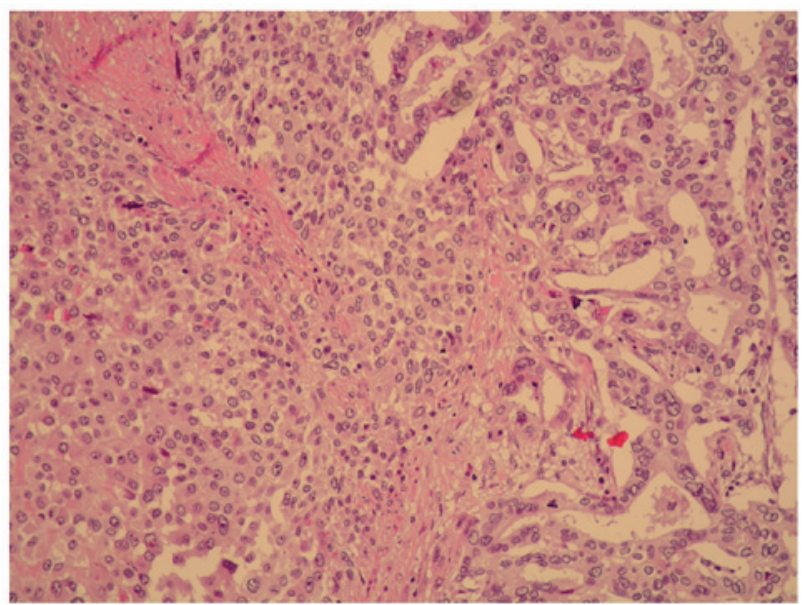

Figure 2. The sarcomatoid component is on the left section of the image and the adenocarcinoma is on the right section of the image.

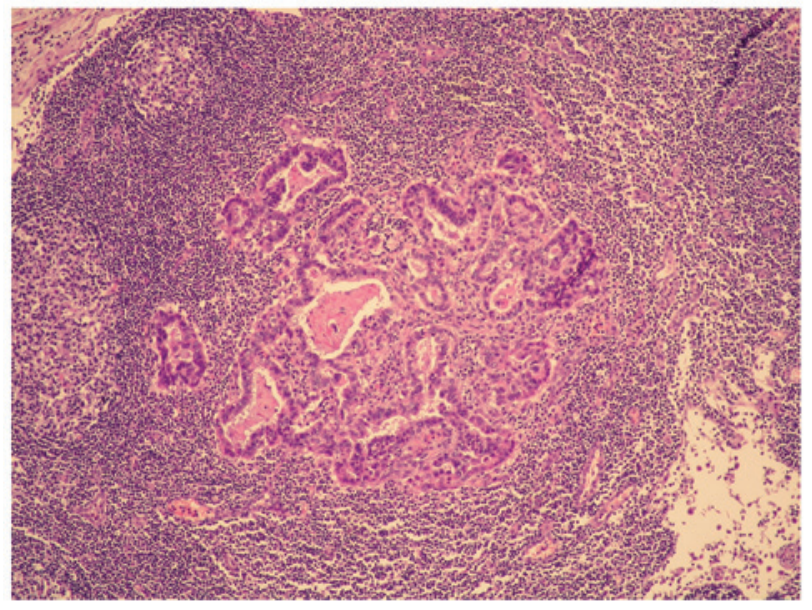

Figure 3. A lymph node with metastases of the carcinomatous component.

by the tumor. Microscopically, the tumor was composed of moderately differentiated adenocarcinoma and poorly differentiated sarcoma with a high mitotic index and necrotic areas (Figs. 1 and 2).

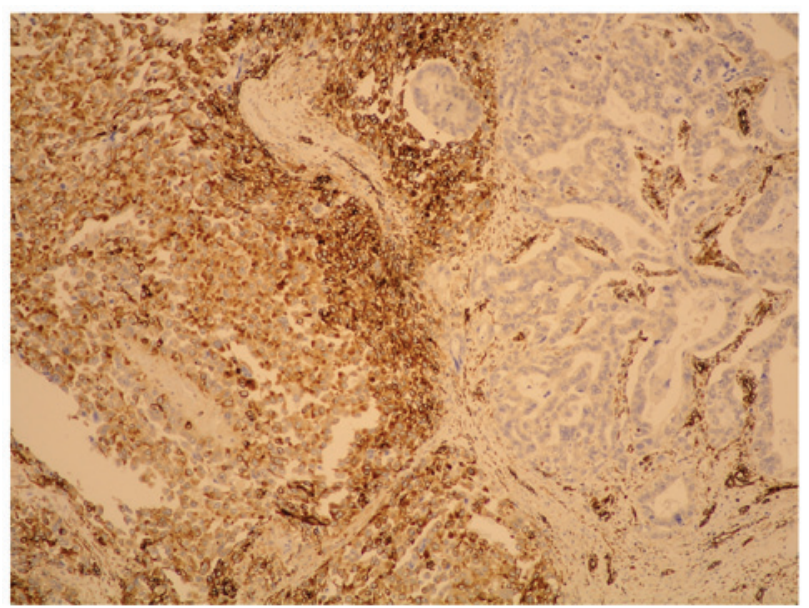

Figure 4. Immunohistochemistry shows intense positivity for vimentin in the sarcomatoid component.

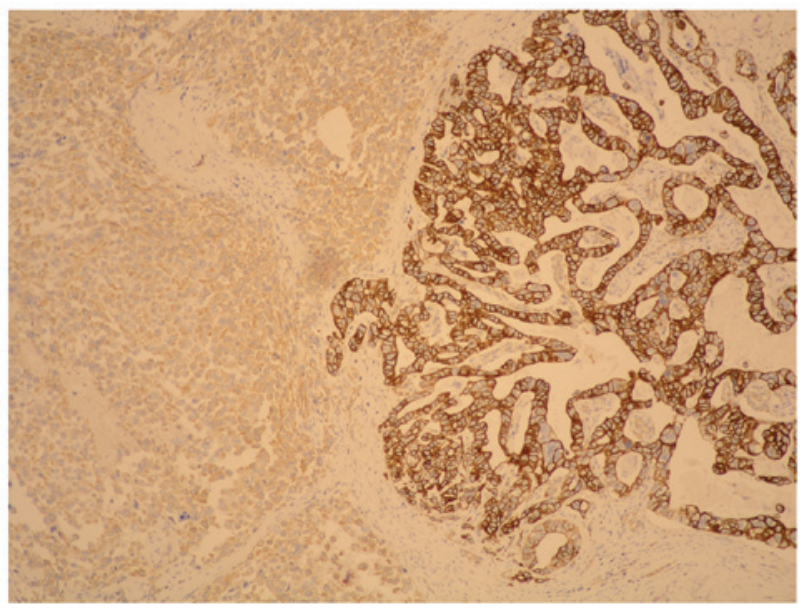

Figure 5. Positivity for CD-57 in the carcinomatous component.

The carcinoma component exhibited a positive reaction to pan-cytokeratin whereas fusiform cells showed positive reactions to vimentin: HHF35; CD56; EMA (weak); desmin (singular component) and negative reactions to cytokeratin, actine, caldesmon, CD-34, S-100 and cromogranin; synaptophysin, CD-57 and c-kit. These immunohistochemical findings led to a diagnosis of gastric carcinosarcoma (Figs. 4 and 5).

The tumor was found to have infiltrated the perivisceral fat and peripancreatic areas. There was neoplastic vein thrombosis of the splenic hilium and, for 2 of 13 regional nodes, metastases were present (Fig. 3). These metastases belonged only to the carcinoma component (pTNM classification was: T4 N1 G3 R0). The post-operative course was unremarkable. Due to poor general health conditions, the patient did not undergo administration of chemotherapy. The patient succumbed to the disease approximately 4 months later.

\section{Discussion}

Carcinosarcoma is defined by the WHO as 'a malignant tumor composed of intimately mixed epithelial and mesenchymal elements of a type ordinarily found in malignancies 


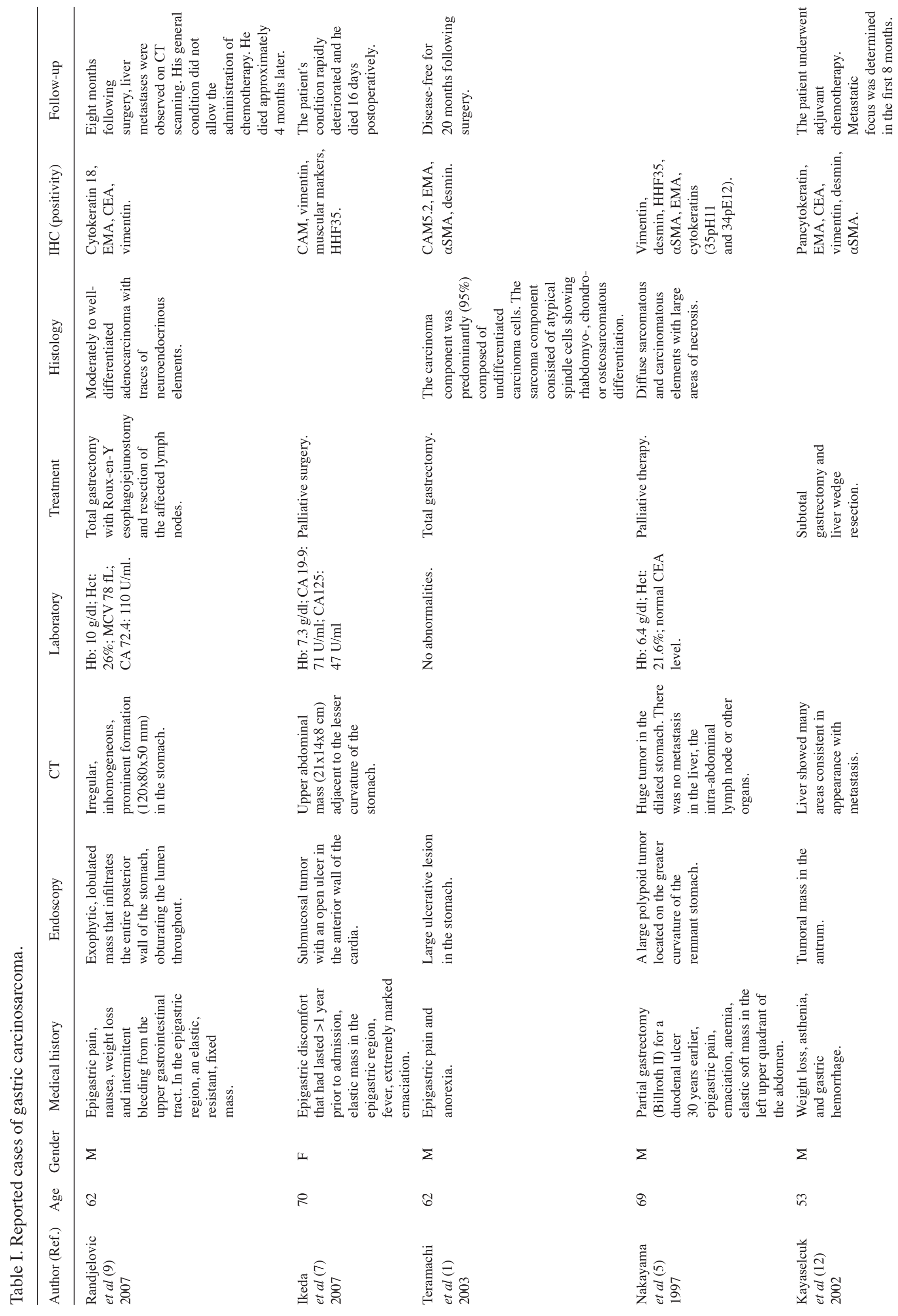




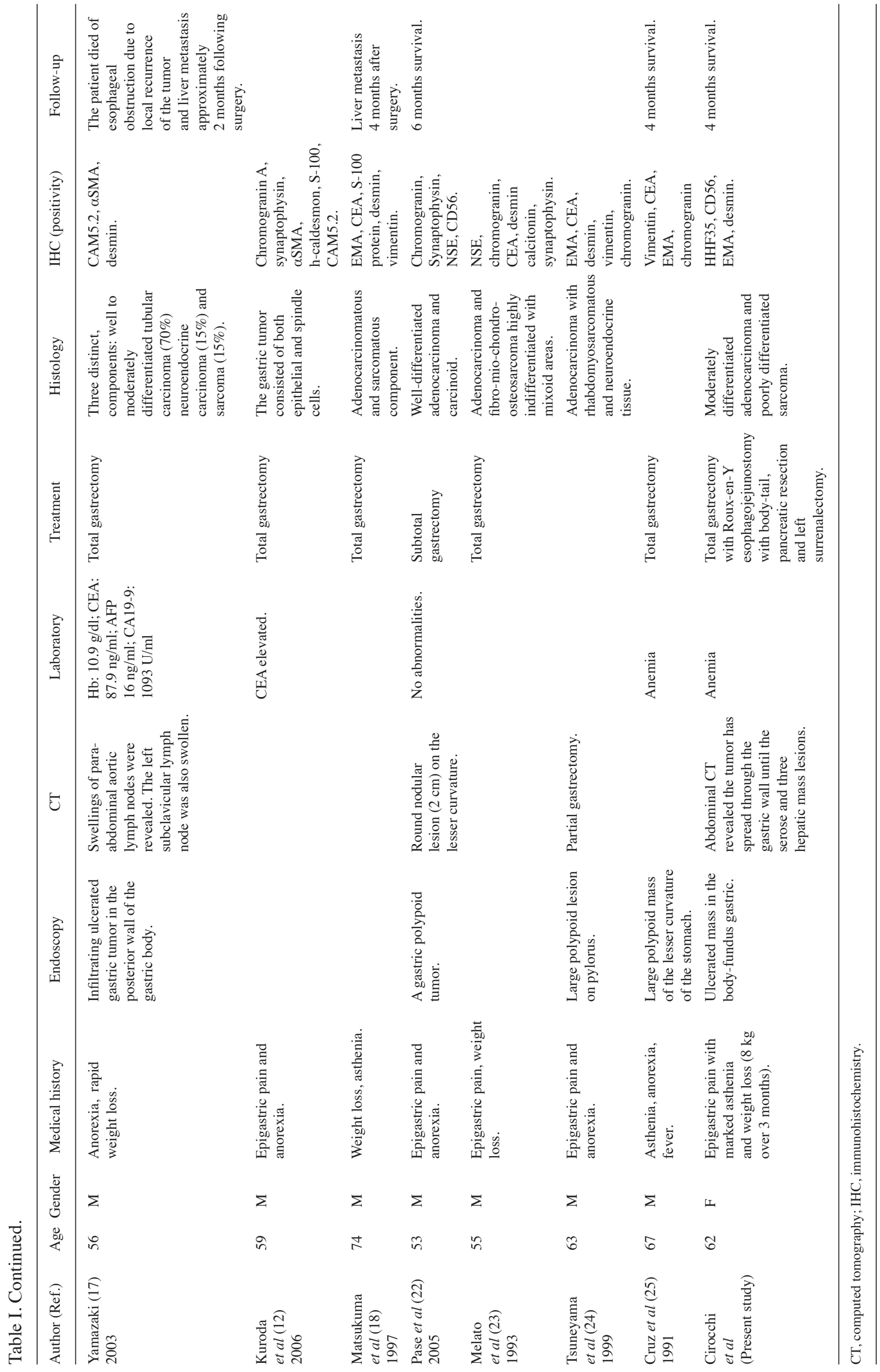


of adults'. This definition is based on traditional histological findings (4). The localizations of this tumor are widespread.

The most common site of origin for these tumors is the uterus. Several other organs such as the salivary gland, thyroid gland, breast, gallbladder, esophagus, stomach, small and large intestine, pancreas, urinary system and the prostate gland may also be affected by this type of tumor.

Localization in the stomach has been less frequently reported (5); male (M) gender is more affected than female (F) $(\mathrm{M}: \mathrm{F}=2.8: 1)$. These data are considerably different from that of other locations where localization to other sites occurs more frequently in women (i.e., gallbladder $\mathrm{M}: \mathrm{F}=1: 3.25$ ).

The median age of patients affected by gastric carcinosarcoma was 62 years (range, 29-80), slightly lower than other localizations in which the most frequent onset age is the geriatric age (6). The youngest patient was 29 years old and this case was originally reported by Saito in 1916 [described by Ashida et al (7)], while the oldest patient was 80 years old, as reported by Ooi in 1982 (8). The median age of male and female patients was 60.9 and 61.3 years, respectively.

The dimensions of this type of tumor calculated on data from 33 reviewed cases and our case report range in size from $4 \mathrm{~cm}$ [Cho et al (9)] to $15 \mathrm{~cm}$ [Saito, described in (7)]. The median dimension of the tumors was $9 \mathrm{~cm}$. According to the macroscopic pattern of growth and particularly in relation to the gastric wall, carcinosarcoma has been classified into three types (10): i) a predominantly intramural infiltration; ii) a predominantly extramural mass; iii) a predominantly intramural mass with exophytic or crater-shaped growth.

Microscopically, carcinosarcoma is classified into two types: true carcinosarcoma and false carcinosarcoma or so-called sarcomatoid carcinoma. Most of the reviewed cases were polypoid ( 20 cases) or ulcerated (19 cases) in appearance. This type of tumor, as with all visceral carcinomas, tends to develop rapidly, appearing similar to an endophytic polypoid mass. It can arise from all areas of the stomach. Cancer does not occur more frequently in any one area. The exact histogenesis remains controversial and remains unknown. However, some authors have proposed two hypotheses (11). The first is the biclonal origin hypothesis that supports the collision tumor theory, according to which the carcinosarcoma originates from two different tumor cell clones. The second is the monoclonal origin hypothesis, whereby the carcinosarcoma may originate from a stem cell that is capable of undergoing both epithelial and mesenchymal differentiation.

In most cases, no specific symptoms of carcinosarcoma were reported and it was the epiphenomenon of locally advanced gastric cancer: asthenia, epigastric pain, dysphagia and vomiting. However, the occurrence of hematemesis and melena were infrequent (12). A mass in the epigastric region is frequently revealed on physical examination. Endoscopic examination is the gold standard in diagnosis as is contrastenhanced CT in the staging of the disease. However, clinical symptoms of carcinosarcomas do not differ from gastric adenocarcinomas, and a discriminating diagnosis is endoscopically or radiologically impossible. Furthermore, only an epithelial or sarcomatous component of the tumor may be observed in small endoscopic biopsies (13).

In $89 \%$ of patients a surgical procedure was performed. In most cases curative surgery was performed. In some rare cases palliative surgery was carried out to restore intestinal continuity or cytoreductive surgery to remove a mass necrosis (14).

The most frequent surgical procedure performed was total gastrectomy, which was often carried out on principle and not of necessity (the tumor had invaded the majority of the organ and/or perivisceral structures, or prior gastrectomy for peptic ulcer disease).

Splenectomy and partial pancreatectomy were not performed on principle but only as a necessity to intervene in patients with tumors that had invaded the surrounding structures. When feasible, resection of liver metastasis was performed at the same time as gastrectomy (15).

In the past, the diagnosis of carcinosarcoma was obtained by conventional histology. The first association between traditional histology and immunohistochemistry in the diagnosis of carcinosarcoma was in a case report in 1988 (16). A third neuroendocrin component has been identified in certain cases in addition to the carcinoma and sarcoma components

CEA, EMA, pancreatin, chromogranin A, CD56 and synaptophysin staining are highly specific markers used to identify carcinomatous components, whereas desmin, vimentin and $\alpha$-smooth muscle/sarcomeric actin show affinity for the sarcomatous elements (17).

A differential diagnosis between GIST and mesotelioma is crucial. GIST often occurs as a large intra-abdominal tumor and sometimes consists of spindle-shaped or epithelioid cells. However, carcinosarcoma shows no immunoreactivity for CD117 or CD34 and it does not demonstrate papillary or glandular structures on H\&E sections, such as those usually observed in malignant mesotheliomas. It also tests negative for mesothelial cell markers, such as calretinin.

In all reviewed cases the mean survival period was extremely poor, approximately 6.5 months excluding the four major (up to 2 years) and minor survivals (less than 30 days). There was no difference in the survival of patients with gastric cancer where the neuroendocrin component was present. Overall tumor recurrence in the first postoperative year was greater than $50 \%$.

From the review of the literature it was not possible to identify any prognostic factor because only a few cases had a survival of longer than 12 months: i) in the case report described by Ashida et al (7), the patient survived 7 years; ii) in that by Tominaga [described in (7)], the patient survived 5 years; iii) in that by Kyogoku et al (18), the patient survived 3 years; iv) in that by Kumagai et al (19), the patient survived 2 years; v) in the one by Teramachi et al (1) the patient survived 20 months; and vi) in the case report described by Kitamura (20), the patient survived for 1 year (Table I).

The most common site of recurrence is the liver where metastases occur immediately after surgery $(17,21)$. In the present case report, liver metastases originate from the adenocarcinoma component.

In conclusion, we reported a case of carcinosarcoma and the procedure for achieving a definitive diagnosis. The simultaneous presence of epithelial and mesenchymal elements in a gastric tumor is a rare event, found almost exclusively in areas with high incidence of gastric cancer and with only few cases reported in literature. Carcinosarcoma of the stomach is a rare malignant tumor of often unclear etiology and pathogenesis. At present, the gold standard for definitive diagnosis is based on immunohistochemical staining of endoscopic biopsy or 
surgical findings. Radical gastrectomy is the treatment of choice when feasible even if the tumor has rapid growth and malignant potential. However, the recurrence of this type of tumor may be expected within the first postoperative year. Therefore, more effective diagnostic techniques should be identified to improve patient survival.

\section{Acknowledgements}

The authors thank Professor Angelo Sidoni and Dr Marta Sbaraglia of the Pathological Anatomy Unit, University of Perugia, for the histopathological analysis.

\section{References}

1. Teramachi K, Kanomata N, Hasebe T, Ishii G, Sugito M and Ochiai A: Carcinosarcoma (pure endocrine cell carcinoma with sarcoma components) of the stomach. Pathol Int 53: 552-556, 2003.

2. Khan AR: Sarcomatoid carcinoma of the stomach with heterologous elements. Ann Saudi Med 19: 135-136, 1999.

3. Solerio D, Ruffini E, Camandona M, Raggio E, Castellano I and Dei Poli M: Carcinosarcoma of the esophagogastric junction. Tumori 94: 416-418, 2008.

4. Maiorana A, Fante R, Maria Cesinaro A and Adriana Fano R: Synchronous occurrence of epithelial and stromal tumors in the stomach: a report of 6 cases. Arch Pathol Lab Med 124: 682-686, 2000.

5. Nakayama Y, Murayama H, Iwasaki H, Iwanaga S, Kikuchi M, Ikeda S, Okada M, Iizuka Y and Iwashita A: Gastric carcinosarcoma (sarcomatoid carcinoma) with rhabdomyoblastic and osteoblastic differentiation. Pathol Int 47: 557-563, 1997.

6. Guerra Bautista JA, Ibáñez Delgado F, Hernández de la Torre Bustillo JM and Alcántara Gijón F: Gastric carcinosarcoma. Rev Esp Enferm Dig 98: 146-147, 2006.

7. Ashida K, Wamata T, Sugesawa A, Miyano Y, Iwai N and Tani H: A case of so-called carcinosarcoma of the stomach. J Jpn Surg Assoc 59: 702-706, 1998.

8. Ooi A, Okada Y, Nakanishi I and Nakajima Y: A case of so-called carcinosarcoma of the stomach (in Japanese). Jpn J Cancer Clin 28: 1300-1304, 1982.

9. Cho KJ, Myong NH, Choi DW and Jang JJ: Carcinosarcoma of the stomach. A case report with light microscopic, immunohistochemical, and electron microscopic study. APMIS 98: 991-995, 1990.
10. Jang SM, Jang SH, Min KW, Na W, Jun YJ and Paik SS: A case of gastric carcinosarcoma with neuroendocrine and smooth muscle differentiation. Korean J Pathol 44: 87-91, 2010.

11. Randjelovic T, Filipovic B, Babic D, Cemerikic V and Filipovic B: Carcinosarcoma of the stomach: a case report and review of the literature. World J Gastroenterol 13: 5533-5536, 2007.

12. Kuroda N, Oonishi K, Iwamura S, Ohara M, Hirouchi T, Mizumo K, Miyazaki E and Enzan H: Gastric carcinosarcoma with neuroendocrine differentiation as the carcinoma component and leiomyosarcomatous and myofibroblastic differentiation as the sarcomatous component. APMIS 114: 234-238, 2006.

13. Kikuyama R, Tanaka K, Tano S, et al: A case of gastric carcinosarcoma. Endoscopy 41: e220-e221, 2009.

14. Ikeda Y, Kosugi S, Nishikura K, et al: Gastric carcinosarcoma presenting as a huge epigastric mass. Gastric Cancer 10: 63-68, 2007.

15. Kayaselcuk F, Tuncer I, Toyganozu Y, et al: Carcinosarcoma of the stomach. Pathol Oncol Res 8: 275-277, 2002.

16. Siegal A, Freund U and Gal R: Carcinosarcoma of the stomach. Histopathology 13: 350-353, 1988.

17. Yamazaki K: A gastric carcinosarcoma with neuroendocrine cell differentiation and undifferentiated spindle-shaped sarcoma component possibly progressing from the conventional tubular adenocarcinoma; an immunohisto-chemical and ultrastructural study. Virchows Arch 442: 77-81, 2003.

18. Kyogoku M, Okukubo T and Aoki S: An autopsy case of carcinosarcoma which originated in the stomach. Gann 51: 278-279, 1960.

19. Kumagai K, Kawai K, Kusano H, Matsuo K, Irie J, Tsuchiyama H and Aridome Y: A case of so-called carcinosarcoma of the stomach. Gan No Rinsho 30: 1931-1936, 1984.

20. Kitamura S: Study on carcinosarcoma of stomach. Gann 41: 15-27, 1950.

21. Matsukuma S, Wada R, Hase K, Sakai Y, Ogata S and Kuwabara N: Gastric stump carcinosarcoma with rhabdomyosarcomatous differentiation. Pathol Int 47: 73-77, 1997.

22. Pase F, Galassi A, Tormen D, Missaglia C, Petrelli G and D'Amore ES: [Composite tumour of the stomach: a case report and review of the literature]. Chir Ital 57: 99-102, 2005.

23. Melato M, Bucconi S, Grillo BP, Angelucci D, Di Stefano P and Natoli C: Carcinosarcoma and separate neuroendocrine malignant tumor of a malignancy promoter, the gastric stump. Anticancer Res 13: 2485-2488, 1993.

24. Tsuneyama K, Sasaki M, Sabit A, Yokoi K, Arano Y, Imai T, et al: A case report of gastric carcinosarcoma with rhabdomyosarcomatous and neuroendocrinal differentiation. Pathol Res Pract 195: 93-97, discussion 8, 1999.

25. Cruz JJ, Paz JI, Cordero M, Martin J and del Mar Abad M: Carcinosarcoma of the stomach with endocrine differentiation. A case report. Tumori 77: 355-357, 1991. 